\title{
Minimierende und maximierende Vernehmungstaktiken: Risiko falscher Geständnisse und sozialpsychologische Wirkmechanismen
}

\author{
Teresa Schneider ${ }^{1,2}$ iD $\cdot$ Lennart May ${ }^{3}$ iD \\ Eingegangen: 2. März 2021 / Angenommen: 8. Juni 2021 / Online publiziert: 19. Juli 2021 \\ ○ Der/die Autor(en) 2021
}

\section{Zusammenfassung}

Einzelberichte deuten auf die Verwendung von Vernehmungstaktiken in Deutschland hin, die die Schwere der Tat und die Konsequenzen eines Geständnisses herunterspielen (Minimierung) und die vorhandenen Beweise und die Schwere der Konsequenzen von bestreitenden Aussagen übertreiben (Maximierung). Forschungsarbeiten zeigen jedoch, dass solche Taktiken die Rate falscher Geständnisse erheblich erhöhen. Es wird im vorliegenden Beitrag argumentiert, dass Minimierungsund Maximierungstaktiken darauf abzielen, eine Aussagemotivation bei Beschuldigten (Zwischenziel) herzustellen, die in eine Geständniserlangung übergehen soll (Hauptziel). Diese Annahme gründet auf einer vorgenommenen Systematisierung und Abgrenzung einzelner Vorgehensweisen dieser Taktiken. Außerdem erklärt der Beitrag kursorisch die sozialpsychologischen Wirkungsweisen dieser Taktiken auf Geständnisentscheidungen. Dies soll helfen, zukünftig Wirkungsweisen einzelner Vorgehensweisen differenzierter wissenschaftlich zu untersuchen, besser zu verstehen und theoretisch zu begründen.

Schlüsselwörter Befragung $\cdot$ Reid-Technik $\cdot$ Beschuldigte $\cdot$ Aussagemotivation $\cdot$ Geständnisentscheidung

\section{Minimization and maximization: risk of false confessions and social psychological mechanisms}

\begin{abstract}
Anecdotal evidence suggests the use of interrogation/interview tactics in Germany that downplay the seriousness of a crime and the consequences of a confession (minimization) and exaggerate the available evidence and the severity of the consequences of denials (maximization). However, research shows that such tactics increase the rate of false confessions in particular. We argue that minimization and maximization aim to establish motivation in suspects to make a statement (intermediate goal), which should lead to a confession (main goal). This assumption is based on a systematization and differentiation of individual procedures of these tactics. In addition, the article explains the social psychological mechanisms of these tactics on confession decisions. This can help to scientifically examine the effects of such distinct tactics in a more sophisticated way and to explain their effects theoretically.
\end{abstract}

Keywords Interrogation $\cdot$ Interview $\cdot$ Reid technique $\cdot$ Suspects $\cdot$ Statement

Dieser Beitrag basiert auf einer Hausarbeit, die im Rahmen des postgradualen Masterstudiengangs Rechtspsychologie an der Psychologischen Hochschule Berlin (PHB) von Teresa Schneider erstellt wurde.

\section{Teresa Schneider}

teresa.schneider@jura.uni-marburg.de

1 Institut für Kriminalwissenschaften, Fachbereich Rechtswissenschaften, Philipps-Universität Marburg, Universitätsstr. 6, 35032 Marburg, Deutschland
Ein Geständnis ${ }^{1}$ wird als Königin der Beweise angesehen und hat einen größeren Einfluss auf die Schuldeinschätzung als andere Beweismittel (Kassin und Neumann 1997). Dies ist riskant, da unter bestimmten Bedingungen auch unschuldige Personen Geständnisse ablegen (z.B. Scherr et al. 2020). So gestehen Beschuldigte z.T. freiwillig falsch,

\footnotetext{
${ }^{1}$ Unter einem Geständnis versteht man eine schriftliche oder mündliche Aussage von strafrechtlich selbstbelastenden Informationen (Drews 2012).
} 
ohne dass die Polizei einen unmittelbaren Einfluss darauf hat (z.B. um eine andere Person zu schützen; Schneider et al. 2021; Volbert et al. 2019), aus strategischen Gründen (z.B. um die Verfolgung einer tatsächlich begangenen schwereren Straftat zu verhindern; Gubi-Kelm et al. 2020) oder aufgrund einer polizeilichen Vernehmung (Gudjonsson 2003). Falsche Geständnisse können die weiteren Ermittlungen und strafrechtlichen Entscheidungen beeinflussen. So zeigten Analysen realer Fälle, dass vernehmungsbedingte falsche Geständnisse andere scheinbar unabhängige Beweise schädlich beeinflussen (z. B. kriminaltechnisch gewonnene Erkenntnisse oder Personenidentifizierungen) und zu Fehlurteilen führen können (Kassin et al. 2012).

Forschungsarbeiten $\mathrm{zu}$ vernehmungsbedingten falschen Geständnissen fokussierten sich in der Vergangenheit v.a. auf zwei Gruppen von Taktiken: Minimierung und Maximierung (z.B. Kassin und McNall 1991). Gemein haben diese Taktiken, dass sie explizit auf die Erlangung von Geständnissen abzielen. Minimierungstaktiken sollen dabei die Schwere der Tat und die Konsequenzen eines Geständnisses herunterspielen. Bei Maximierungstaktiken kommt es hingegen zu einer Übertreibung und Betonung der vorhandenen Beweise und der Schwere der Konsequenzen von Bestreiten (siehe unten für eine ausführliche Beschreibung der Taktiken). Solche Vernehmungstaktiken erhöhen nicht nur die Wahrscheinlichkeit wahrer, sondern insbesondere auch falscher Geständnisse (z. B. Narchet et al. 2011). Dies ist besonders problematisch, da nicht unmittelbar an der Vernehmungsinteraktion beteiligte Personen (z.B. Staatsanwält*innen, Richter*innen) das Risiko von Minimierung unterschätzen (Kassin und McNall 1991).

Der vorliegende Beitrag beschäftigt sich mit Minimierungs- und Maximierungstaktiken und folgenden Fragen: a) Werden diese Taktiken in Deutschland angewendet? b) Welche Forschungsergebnisse gibt es zum Einfluss dieser Taktiken auf das Geständnisverhalten? c) Wie können die einzelne Vorgehensweisen von Minimierung und Maximierung abgegrenzt und systematisch geordnet werden? und d) Welche sozialpsychologischen Wirkmechanismen liegen Geständnissen zugrunde, und wie können Minimierungs- und Maximierungstaktiken diese beeinflussen? Dieser Aufbau orientiert sich daran, dass insbesondere amerikanische Psycholog*innen Minimierung- und Maximierungstaktiken zunächst in Vernehmungsmanualen und der Praxis beobachteten und daraufhin begannen, sie empirisch zu untersuchen (z.B. Kassin 2007; Kassin und McNall 1991).

\section{Anwendung von Minimierungs- und Maximierungstaktiken}

Internationale Vernehmungsmanuale (z.B. Inbau et al. 2013) und Feldstudien (z.B. Leo 1996) weisen darauf hin, dass Polizist*innen in Minimierungs- und Maximierungstaktiken unterrichtet werden und diese auch praktisch anwenden (Kassin 2007). Häufig kommt es zur Anwendung solcher geständnisorientierten Taktiken, wenn Vernehmende subjektiv von der Schuld der Beschuldigten überzeugt sind und aufgrund einer unvollständigen Beweiskette diese zu Aussagen und Geständnissen motivieren wollen (sog. Tunnelblick; Findley und Scott 2006; Volbert und May 2016). Dabei ignorieren sie oftmals das Risiko, dass sie durch Minimierung und Maximierung falsche Geständnisse erlangen können und es dadurch zu einer falschen Bestätigung ihrer Schuldüberzeugung kommen kann (Leo 2020; May 2021; Meissner et al. 2010). Ihre Fehleinschätzungen werden in solchen Fällen nicht korrigiert, sondern vielmehr verstärkt (Narchet et al. 2011). Nachfolgend wird exemplarisch auf drei solcher Vernehmungsmanuale bzw. -techniken eingegangen, die auch für die deutsche Polizeipraxis relevant scheinen. Jedoch können solche Taktiken in der Praxis auch unabhängig davon, ob Vernehmende solche Manuale gelesen haben, Anwendung finden. Denn es ist denkbar, dass diese Taktiken auch implizit und intuitiv ohne die entsprechende Benennung der Taktiken als Minimierung oder Maximierung angewendet werden. Im Einklang damit empfiehlt die deutschsprachige Vernehmungsliteratur beispielsweise, dass Vernehmende Beschuldigten aufzeigen sollen, dass sich Kooperationsbereitschaft strafmildernd auswirken und sie auch die befürchteten sozialen Folgen minimieren kann (Minimierung; Heubrock und Donzelmann 2010). Bei weiterhin unkooperativen Beschuldigten sollen Vernehmende hingehen auf soziale Folgen von Pressemitteilungen hinweisen (Maximierung).

\section{Reid-Technik}

Die Reid-Technik (Inbau et al. 2013) ist in den USA stark verbreitet und basiert weitestgehend auf 2 Prozessen: (1) den Aussagewiderstand und das Bestreiten ,brechen“ und (2) den „Geständniswunsch“ vergrößern (Gudjonsson 2003). In der ersten Phase der Reid-Technik findet dazu eine Art informelles Vorgespräch statt. Hier sollen Vernehmende Hintergrundinformationen über die Beschuldigten sammeln, die sie später gegen sie verwenden können. AuBerdem sollen sie durch Beobachten von verbalem und nonverbalem Verhalten der Beschuldigten deren Schuld einschätzen (,,behavior analysis interview“). Dieses Vorgehen missachtet jedoch umfangreiche Forschungsarbeiten, wonach Lügen nicht anhand dieser Indikatoren erkennbar sind (z. B. DePaulo et al. 2003; Vrij et al. 2019), und Ex- 
perimente, die die Ineffizienz dieser Phase belegen (Kassin und Fong 1999; Vrij et al. 2006).

Bei positiver Schuldeinschätzung folgt in der zweiten Phase die "formelle“ Vernehmung, die in 9 Stufen gegliedert ist. In einzelnen dieser Stufen werden sowohl Minimierungs- als auch Maximierungstaktiken verwendet. Beispielsweise werden in der zweiten Stufe (,theme development“) explizit Minimierungstaktiken aufgeführt, um die Aussagemotivation bei Beschuldigten herzustellen. Das Reid-Manual gibt hier u.a. vor, dass Vernehmende moralisch akzeptable Erklärungen für die Tat vorgeben und andere Personen für die Tat beschuldigen sollen. Maximierungstaktiken werden in der dritten (Bestreiten überwinden) und vierten Stufe (Einwände beseitigen) beschrieben: Vernehmende sollen Beschuldigten mit absoluter Sicherheit sagen, dass sie die Tat begangen haben und bestreitende Angaben nicht akzeptieren.

Die Reid-Technik und ihre risikoreichen Befragungstaktiken werden in der internationalen Forschung deutlich kritisiert und abgelehnt (z. B. Gudjonsson 2003; Kassin et al. 2010). Auch die Bundesregierung (2014) bewertet diese Technik in Bezug auf $\S 136$ a StPO kritisch. Trotz allem wurde sie in der Vergangenheit in Deutschland unterrichtet und vermutlich auch in der Praxis angewendet (Lindner 2002). So führte beispielsweise die bayerische Polizei in den Jahren 2001 und 2002 insgesamt 6 Fortbildungsseminare zur Reid-Technik durch (Bayerischer Landtag 2014). In einer Befragung von erfahrenen Kriminalbeamt*innen gaben $42 \%$ an, an einer Ausbildung in der Reid-Technik teilgenommen zu haben (Litzcke und Klossek 2008 zitiert nach Hermanutz und Litzcke 2012). Auch ergeben sich Hinweise, dass die Polizei in Einzelfällen zumindest Teile dieser Technik anwendete und falsche Geständnisse erlangte (z. B. Jung und Lemmer 2013; May 2019; Rick 2012). Aufgrund fehlender systematischer Untersuchungen ist jedoch unklar, inwiefern die Reid-Technik aktuell in Deutschland angewendet wird.

\section{RPM-Methode}

Die ebenfalls aus den USA stammende RPM-Methode ist als Ableger der Reid-Technik anzusehen (Napier und Adams 1998). Die Abkürzung RPM steht für Rationalisierung, Projektion und Minimierung und basiert auf der Annahme, dass Täter*innen durch diese Abwehrmechanismen ihre Tat rechtfertigen. Vernehmende sollen wiederum Rationalisierung, Projektion und Minimierung nutzen, um Täter*innen Rechtfertigungsstrategien für die Tat anzubieten (Artkämper und Schilling 2018) und es ihnen zu erleichtern, über die Tat zu sprechen (Hermanutz und Litzcke 2012; Napier und Adams 1998).

Die Taktik, die explizit als Minimierung bezeichnet wird, spielt die Tat und die Tatbeteiligung herunter und bagatel- lisiert diese. Beispielsweise soll ein Tötungsdelikt nicht als Mord, sondern als Unfall beschrieben werden (z. B. „Unfälle wie diese passieren einfach"; Artkämper und Schilling 2018). Doch auch Rationalisierung und Projektion sind den Minimierungstaktiken zuzuschreiben. Bei der Rationalisierung sollen die Vernehmenden den Beschuldigten plausible Erklärungen für die Tat liefern und sich verständnisvoll und empathisch zeigen (z.B. „In dieser [Ausnahme]Situation haben Sie völlig normal gehandelt“; Artkämper und Schilling 2018). Bei der Projektion sollen Vernehmende die Schuld für die Tat auf andere Personen oder auf die Situation schieben (z. B. ,Jugendliche können schwierig zu handhaben sein"; Napier und Adams 1998).

Die deutschsprachige Literatur beschreibt, dass die RPM-Methode mit der StPO unvereinbar sei, wenn es ausschließlich um die Erlangung von Geständnissen gehe (z.B. Artkämper und Schilling 2018; Hermanutz und Litzcke 2012). Jedoch seien bestimmte Taktiken bei richtiger Zielsetzung nicht verkehrt, da sie den Täter*innen helfen würden, über das Geschehen zu sprechen, wobei die Taktiken auch nicht zur Doktrin werden dürften. Diese Annahme vernachlässigt jedoch die eingangs dargelegte Überlegung: Wenn Vernehmende bei fehlender eindeutig belastender Beweislage lediglich subjektiv von der Schuld der Beschuldigten überzeugt sind, wird bei der Anwendung von Minimierung und Maximierung versucht, die Beschuldigten zu einer Aussage zu motivieren und gleichzeitig die Gefahr falscher Geständnisse missachtet. Belastbare Daten, inwiefern die RPM-Methode auch in Deutschland angewendet wird, liegen nicht vor. In der persönlichen Kommunikation wurde jedoch mitgeteilt, dass die RPMMethode bei der bayerischen Polizei in der Vergangenheit gelehrt wurde.

\section{Mr.-Big-Technik}

Die Mr.-Big-Technik wurde in Kanada für den Kontext verdeckter Ermittlungen entwickelt (z.B. Smith et al. 2010; van Toor und Horselenberg 2020) und lässt sich in 4 Bereiche unterteilen: Zunächst sammeln Ermittelnde bei verdeckten Maßnahmen Informationen zu den Verdächtigen (z.B. zur Arbeitssituation, Familie, Freizeitgestaltung; Luther und Snook 2016). Diese Informationen nutzen sie dann, um sich erfolgreich bei den Verdächtigen „einzuschleusen“" (Luther und Snook 2016). Die Kontaktaufnahme kann dabei auf sehr unterschiedliche Arten erfolgen (z.B. in polizeilichem Gewahrsam, in Entzugskliniken oder über den Arbeitsplatz), wobei die Ermittelnden anschließend viel Zeit mit den Zielpersonen verbringen, um ein freundschaftliches Verhältnis vorzuspielen (z.B. Einladungen in Restaurants, Bars und Nachtclubs). In der dritten Phase beziehen die Ermittelnden die Zielpersonen in kriminelle Handlungen ein und bezahlen sie sehr großzügig (Luther und Snook 2016). 
Normalerweise bekommen sie zunächst kleinere Aufgaben (z.B. Überwachungsaufgaben, Botengänge), die im weiteren Verlauf schwieriger, verantwortungsvoller und besser bezahlt sind (z. B. gewalttätiges Schulden eintreiben oder Verkaufen von Schmuggelware wie Drogen und Waffen). Weiterhin sind die Verdächtigen auch in soziale Aktivitäten eingebunden (z.B. Besuche in Bars, Stripclubs, hochklassigen Hotels und Restaurants und in nationale und internationale Ausflüge). Dies soll den Zielpersonen den extremen Wohlstand, die Macht und die Einflussmöglichkeiten der Organisation demonstrieren. In der vierten Phase kommt es zu einem Treffen der Verdächtigen mit dem angeblichen Chef der Organisation „Mr. Big“ (Luther und Snook 2016; Smith et al. 2010). Vorgegebener Grund des Treffens ist eine Beförderung der Verdächtigen, im Zuge dessen sie implizit oder explizit zu einem Geständnis gebracht werden sollen: Beispielsweise kann den Verdächtigen mitgeteilt werden, dass sie für ihre Aufnahme in die Organisation im Sinne eines Vertrauensbeweises ein bestimmtes Verbrechen oder alle früheren Taten gestehen müssen. Den Zielpersonen kann auch mitgeteilt werden, dass die Organisation ihre Kontakte nutzen werde, um alle Beweise gegen sie loszuwerden (z.B. eine andere Person werde für die Sache gestehen und in Haft gehen). Bei allen Alternativen wird den Verdächtigen jedoch klar vermittelt: Sie müssen gestehen, um Teil der Organisation zu bleiben und den luxuriösen Lebensstil fortführen zu können.

Die Mr-Big-Technik beinhaltet auch Minimierungs- und Maximierungstaktiken. Beispielsweise werden in dem vorgespielten Szenario die positiven Folgen eines Geständnisses hervorgehoben (z. B. Minimierung: Aufstieg in der Organisation) und gleichzeitig die negativen Folgen von Bestreiten betont (z. B. Maximierung: Verlieren des gut bezahlten Jobs innerhalb der Organisation). Nachteile eines Geständnisses sind für Verdächtige nicht erkennbar, da sie davon ausgehen, dass die Ermittelnden selbst kriminell sind und es um den Aufstieg in der kriminellen Organisation geht.

Smith et al. (2009) kommen zu dem Schluss, dass viele der rechtlichen Schutzvorkehrungen einer formellen Vernehmung bei der Mr. Big-Technik fehlen und diese noch stärker auf Manipulation, Täuschung und Druck basiert. Dadurch ist das Risiko, unzuverlässige Informationen (falsche Geständnisse) zu erhalten, bei der Mr-Big-Technik vermutlich noch größer als bei der Reid-Technik. Es gibt keine offiziellen Angaben darüber, inwiefern diese Technik aktuell auch in Deutschland angewendet wird, allerdings gibt es gerichtliche Entscheidungen, die auf das Vorkommen der Mr.-Big-Technik - u. a. Namen - auch in Deutschland hinweisen (z.B. OLG Zweibrücken zu verdeckten Ermittlungen nach der „Cold-Case-Technik“, Urteil vom 26.05.2010, 1 Ws 241/09. NStZ 2011, 113-116).
Die Ausführungen zur Reid-Technik, RPM-Methode und Mr-Big-Technik legen nahe, dass deutschsprachige Arbeiten diese Techniken zwar teilweise kritisieren, jedoch nicht deutlich ablehnen. Außerdem ergaben sich Hinweise, dass mindestens Teile dieser Techniken - und somit auch Minimierung und Maximierung - in der polizeilichen Lehre und Praxis implizit oder explizit angewendet wurden.

\section{Das Risiko falscher Geständnisse}

Allgemein erhöhen Minimierungs- und Maximierungstaktiken die Wahrscheinlichkeit von wahren Geständnissen, aber insbesondere auch von falschen Geständnissen (Meissner et al. 2010). Dadurch führen sie zu einer geringeren Diagnostizität (d.h. Verhältnis von wahren zu falschen Geständnissen) und sind kritisch zu betrachten.

Die Effektivität von Minimierungs- und Maximierungstaktiken lässt sich zuverlässig nur mit Experimenten einschätzen, bei denen die zugrunde liegende Schuld der Versuchspersonen kontrollierbar ist. In einem ersten solchen Experiment untersuchten Russano et al. (2005), welchen Einfluss Minimierungstaktiken und das Anbieten eines Deals auf das Geständnisverhalten haben. Hierzu entwickelten sie das „Cheating“-Paradigma, bei dem die Hälfte der Versuchspersonen zum Schummeln verleitet wurde. Dadurch waren sowohl wahre als auch falsche Geständnisse möglich. ${ }^{2}$ In der Minimierungsbedingung milderte der Versuchsleiter die Schwere des Schummelns ab, indem er Sympathie zum Ausdruck brachte, Entschuldigungen anbot (z. B. „Ich bin sicher, dass Ihnen nicht klar war, was für eine große Sache das war.") und den Teilnehmenden nahelegte, dass es in ihrem Interesse sei, zu kooperieren und das Geständnis zu unterschreiben. In dieser Studie erhöhten Minimierungstaktiken zwar die Rate wahrer Geständnisse, aber eben auch erheblich die Wahrscheinlichkeit falscher Geständnisse. Außerdem zeigte sich eine geringere Diagnostizität im Vergleich zur Bedingung, in der keine Taktiken verwendet wurden. Alle Ergebnisse der hier berichteten Studien im Einzelnen und auch die errechnete Diagnostizität zeigt Tab. 1.

\footnotetext{
${ }^{2}$ Die Versuchspersonen bearbeiteten jeweils gemeinsam mit einer Konföderierten Problemlöseaufgaben. Bestimmte Aufgaben sollten sie individuell lösen, andere hingegen gemeinsam. In der schuldigen Bedingung bat die Konföderierte die Versuchsperson bei einer der individuellen Aufgaben um Hilfe. Wenn die Versuchsperson half, wurde sie als schuldig angesehen. Am Ende des Experiments wurden alle Versuchspersonen beschuldigt, geschummelt (bzw. geholfen) zu haben und aufgefordert, ein Geständnis zu unterschreiben.
} 
Tab. 1 Übersicht der Studienergebnisse zum Einfluss von Minimierung und Maximierung auf die Geständnisrate

\begin{tabular}{|c|c|c|c|}
\hline Autoren & Methode und unabhängige Variablen (UV) & Geständnisrate & Diagnostizität \\
\hline \multirow{4}{*}{$\begin{array}{l}\text { Russano } \\
\text { et al. (2005) }\end{array}$} & \multirow{4}{*}{$\begin{array}{l}\text { Cheating Paradigma } \\
\text { UV: Minimierung, Anbieten eines Deals }\end{array}$} & Keine Taktiken: $46 \%$ wahre, $6 \%$ falsche & 7,67 \\
\hline & & Minimierung: $81 \%$ wahre, $18 \%$ falsche & 4,50 \\
\hline & & Deal: $72 \%$ wahre, $14 \%$ falsche & 5,14 \\
\hline & & Minimierung und Deal: $87 \%$ wahre, $43 \%$ falsche & 2,02 \\
\hline \multirow[t]{2}{*}{$\begin{array}{l}\text { Klaver et al. } \\
(2008)\end{array}$} & \multirow[t]{2}{*}{$\begin{array}{l}\text { ALT-Key-Paradigma } \\
\text { UV: Minimierung und Maximierung, hohe und } \\
\text { niedrige Plausibilität }\end{array}$} & $\begin{array}{l}\text { Hohe Plausibilität } \\
\text { Minimierung: } 70 \% \text { falsche, Maximierung: } 47 \% \\
\text { falsche }\end{array}$ & 1,49 \\
\hline & & $\begin{array}{l}\text { Niedrige Plausibilität } \\
\text { Minimierung: } 23 \% \text { falsche, Maximierung: } 5 \% \\
\text { falsche }\end{array}$ & 4,60 \\
\hline \multirow{4}{*}{$\begin{array}{l}\text { Narchet } \\
\text { et al. }(2011)\end{array}$} & \multirow{4}{*}{$\begin{array}{l}\text { Cheating Paradigma } \\
\text { UV: Ermittlungsverzerrung (Keine; Schuld; } \\
\text { Unschuld) }\end{array}$} & Keine Taktiken: $96 \%$ wahre, $3 \%$ falsche & 32,00 \\
\hline & & Minimierung: $98 \%$ wahre, $22 \%$ falsche & 4,45 \\
\hline & & Maximierung: $92 \%$ wahre, $11 \%$ falsche & 8,36 \\
\hline & & $\begin{array}{l}\text { Minimierung und Maximierung: } 73 \% \text { wahre, } 43 \% \\
\text { falsche }\end{array}$ & 1,70 \\
\hline \multirow[t]{2}{*}{$\begin{array}{l}\text { Horgan et al. } \\
(2012)\end{array}$} & \multirow{2}{*}{$\begin{array}{l}\text { Cheating Paradigma } \\
\text { UV: Minimierung und Maximierung mit Einfluss } \\
\text { auf wahrgenommene Konsequenzen oder nicht }\end{array}$} & $\begin{array}{l}\text { Taktiken mit Konsequenzen: } 82 \% \text { wahre, } 42 \% \\
\text { falsche }\end{array}$ & 1,93 \\
\hline & & $\begin{array}{l}\text { Taktiken ohne Konsequenzen: } 97 \% \text { wahre, } 21 \% \\
\text { falsche }\end{array}$ & 4,58 \\
\hline
\end{tabular}

Klaver et al. (2008) untersuchten mittels des sog. $A L T$ Key-Paradigmas ${ }^{3}$ die Auswirkungen der Kombination von Minimierungs- und Maximierungstaktiken auf die Rate falscher Geständnisse. In der Minimierungsbedingung normalisierten die Versuchsleiter die vorgeworfene Handlung (das Drücken der ALT-/ESC-Taste), bezeichneten sie als Unfall und gaben dem Computer die Schuld. In der Maximierungsbedingung schüchterten die Versuchsleiter hingegen die Versuchspersonen ein und übertrieben die Schwere der vermeintlichen Handlung. Die Wahrscheinlichkeit, ein falsches Geständnis zu unterschreiben, war mehr als 4-mal so hoch, wenn Minimierungstaktiken eingesetzt wurden, im Vergleich zu Maximierungstaktiken. Dieses Ergebnis könnte vermuten lassen, dass Maximierungstaktiken die Rate falscher Geständnisse nicht erhöhen. Dieser Rückschluss kann mit dieser Studie jedoch nicht gezogen werden, da es keine Kontrollgruppe gab und deshalb der isolierte Effekt von Minimierung und Maximierung nicht bestimmbar ist. Außerdem kam es in der Maximierungsbedingung nicht zu einer Übertreibung der vorhandenen Beweise und einer Erklärung, dass Bestreiten zu einer höheren Strafe

\footnotetext{
3 Versuchspersonen tippten Buchstaben auf einer PC-Tastatur ein, die von einem/einer Konföderierten vorgelesen wurden (Kassin und Kiechel 1996). Die Versuchspersonen wurden gewarnt, nicht die ALTTaste (hohe Plausibilitätsbedingung) oder die ESC-Taste (niedrige Plausibilitätsbedingung) zu drücken, da dies zum Absturz des Computers führen würde. Unabhängig vom Verhalten der Versuchspersonen stürzte der Computer nach einer Minute vermeintlich ab, und die Versuchspersonen wurden beschuldigt, die ALT-Taste bzw. ESC-Taste gedrückt zu haben. Daraufhin wurden sie aufgefordert, ein schriftliches Geständnis zu unterschreiben.
}

führen werde. Doch gerade diese Kombination bei der Maximierung erhöht die Wahrscheinlichkeit eines falschen Geständnisses (Kassin und McNall 1991). Es ist deshalb denkbar, dass die Wirkung von Maximierungstaktiken in dieser Studie unterschätzt wurde.

Eine weitere Studie untersuchte, inwiefern Taktiken der Minimierung und Maximierung mit Einfluss auf die wahrgenommenen Konsequenzen eines Geständnisses die Rate falscher Geständnisse erhöhen (Horgan et al. 2012). Die Autoren verwendeten das oben beschriebene Cheating-Paradigma und teilten die verschiedenen Taktiken in 2 Gruppen auf, die die wahrgenommenen Konsequenzen eines Geständnisses beeinflussen oder nicht (Tab. 2). Versuchspersonen legten häufiger ein falsches Geständnis ab, wenn Taktiken mit Einfluss auf die wahrgenommenen Konsequenzen verwendet wurden. Bei wahren Geständnissen zeigte sich ein gegenteiliger Effekt. Bei der Beeinflussung der Konsequenzen zeigte sich außerdem eine geringere Diagnostizität als bei fehlender Thematisierung der Konsequenzen. In dieser Studie wurde jedoch allgemein der Effekt der Beeinflussung der Konsequenzen auf das Geständnisverhalten untersucht, wodurch sich der Effekt nicht isoliert Minimierungsund Maximierungstaktiken zuordnen lässt. Außerdem gab es auch hier keine Kontrollbedingung.

Eine weitere Studie fand, dass eine Schuldüberzeugung der Befragenden zu einer vermehrten Anwendung von Minimierungstaktiken führte und so die Wahrscheinlichkeit falscher Geständnisse erhöhte (Narchet et al. 2011). AuBerdem war sowohl bei Anwendung von Minimierungs- als auch von Maximierungstaktiken die Rate falscher Geständnisse im Vergleich zu einer Kontrollgruppe erhöht. Wurden 
Tab. 2 Einteilung von verschiedenen Taktiken der Minimierung und Maximierung, die die wahrgenommenen Konsequenzen eines Geständnisses beeinflussen oder nicht (nach Horgan et al. 2012)

\begin{tabular}{lll}
\hline & Minimierung & Maximierung \\
\hline Konsequenzen & Betonung des Nutzens der Kooperation & Übertreibung von Konsequenzen \\
& Herunterspielen der Konsequenzen & Gegenseitige Ausspielung von Komplizen \\
& Anbieten von Entschuldigungen & \\
Keine & Herunterspielen der Schwere des Vergehens & \\
Konsequenzen & Sympathie entgegenbringen & Unfreundliches Verhalten \\
& Dem Beschuldigten schmeicheln & Klare Schuldüberzeugung zeigen \\
& Freundliches Verhalten & \\
\hline
\end{tabular}

Die Tabelle von Horgan et al. (2012) wurde von uns ins Deutsche übersetzt

beide Taktiken verwendet, erhöhte sich die Wahrscheinlichkeit eines falschen Geständnisses nochmals und die Diagnostizität war am geringsten (Tab. 1).

Zusammenfassend zeigten die Experimente, dass Maximierungstaktiken, aber insbesondere auch Minimierungstaktiken die Wahrscheinlichkeit falscher Geständnisse deutlich erhöhen. Jedoch ist die Studienlage zu Maximierungstaktiken sehr gering. Eine Studie, die den isolierten Einfluss von Maximierungstaktiken auf das Geständnisverhalten untersucht, ist uns nicht bekannt. Der Einfluss von Minimierungstaktiken wurde zwar in einigen Studien untersucht, jedoch wurde bisher beispielsweise nicht der Frage nachgegangen, ob verschiedene Arten der Minimierungstaktiken einen unterschiedlichen Effekt auf die Geständnisrate haben.

\section{Arten von Minimierungs- und Maximierungstaktiken}

Ein Literaturstudium ergab, dass einschlägige Arbeiten verschiedene Arten von Minimierungs- und Maximierungstaktiken beschreiben (z. B. Inbau et al. 2013; Napier und Adams 1998; Nasher 2015; Senese 2012). Diese Arten der Taktiken haben gemein, dass sie manipulativ sind und in der Vernehmung zunächst darauf abzielen, den Aussagewiderstand ${ }^{4}$ von Beschuldigten aufzulösen (im Sinne eines Zwischenziels), um sie dann zu einem Geständnis „zu bringen“ (im Sinne eines Hauptziels). Die Auflösung des Aussagewiderstands ist somit mit einer Geständnismotivierung gleichzusetzen. Problematisch ist, dass diese Literaturarbeiten offenbar fälschlicherweise davon ausgehen, dass nur schuldige Personen vernommen werden und

\footnotetext{
${ }^{4}$ Laut dem Kriminalistik-Lexikon beschreibt der Aussagewiderstand eine Verhaltensweise einer Aussageperson, die in einer Vernehmung nicht oder falsch aussagt. Der Aussagewiderstand wird von der Widerstandsenergie, der Widerstandsintelligenz und dem Widerstandswillen bestimmt. Er wird durch die Aussagemotivation beeinflusst und zeigt sich im Aussageverhalten (Wirth 2013).
}

Falschaussagen zuverlässig einschätzbar sind. Außerdem dürften Vernehmende, die subjektiv von der Schuld der Beschuldigten überzeugt sind, alles außer einem Geständnis als Aussagewiderstand betrachten (z. B. Bestreiten von Tatvorwürfen, keine Angaben machen; siehe Fußnote 4). Wie oben dargestellt, beleuchten Forschungsarbeiten bisher eher Gruppen von Minimierungs- und Maximierungstaktiken. Im Folgenden wird nun eine Systematisierung einzelner Arten von Minimierung und Maximierung vorgenommen, um einen Rahmen für zukünftige Überlegungen und Forschung zu schaffen.

\section{Minimierungstaktiken}

Mit Minimierungstaktiken sollen Vernehmende durch ein emotional- und inhaltlich-manipulatives Vorgehen den Aussagewiderstand von Beschuldigten auflösen (Zwischenziel) und sie zu einem Geständnis bewegen (Hauptziel). Die verschiedenen Zwischenziele, die sich aus der oben genannten Literatur ableiten lassen, zeigt Abb. 1.

Um zu erreichen, dass Beschuldigte sich sicher fühlen und den Vernehmenden vertrauen, sollen sich diese freundlich, sympathisch und unaufdringlich zeigen (Kassin und McNall 1991). Außerdem sollen sie Beschuldigten Erklärungsmöglichkeiten wie Entschuldigungen und Rechtfertigungen für die Tat anbieten, sodass diese ihre Tatverantwortung gering einschätzen. Diese Erklärungen beschreiben Tatvorgänge, die die psychologische Tatverantwortung und kriminelle Absicht von Beschuldigten herunterspielen, aber eine physische Tatverantwortung implizieren. Beschuldigte sollen diese Erklärungen ,annehmen“ und in ihre Aussage integrieren (Senese 2012). Beispielsweise können Vernehmende den Umständen die Schuld geben (z.B. „In Ihrer Situation hätte ich genau das Gleiche getan“; Inbau et al. 2013), anderen Personen die Tatverantwortung zuschreiben (z. B. „Sie hat sich so aufreizend verhalten und Sie geküsst. Sie hat es doch herausgefordert"; Horgan et al. 2012) oder moralisch akzeptable Erklärungen vorschlagen (z. B. „Sie wollten doch nur Gutes tun und haben deswegen das Geld 
Abb. 1 Zwischenziele von Minimierungstaktiken, die zu einem Geständnis führen sollen
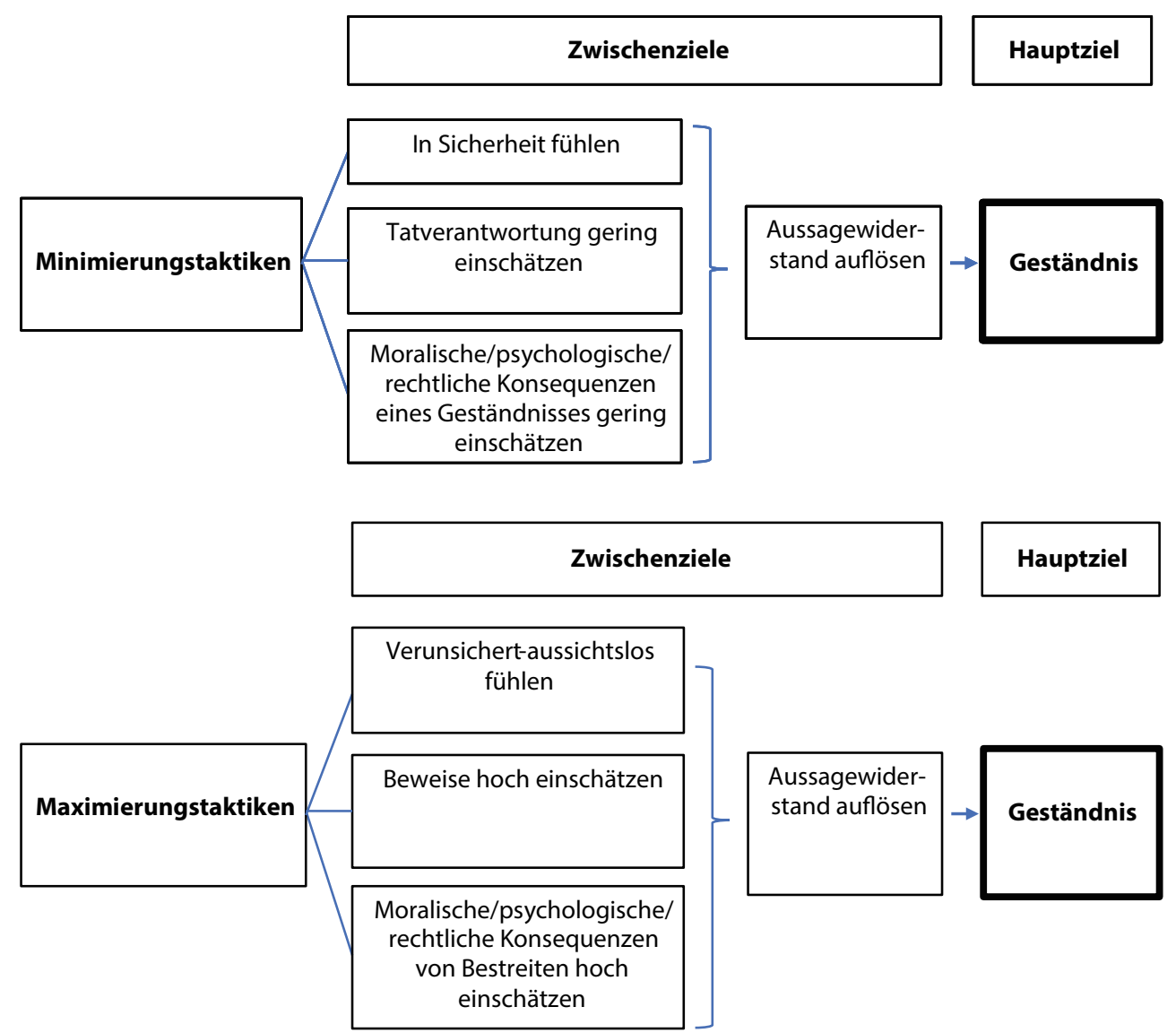

Abb. 2 Zwischenziele von Maximierungstaktiken, die zu einem Geständnis führen sollen
Um zu erreichen, dass Beschuldigte sich verunsichert und aussichtslos fühlen, sollen Vernehmende Beschuldigte wiederholt mit dem Tatvorwurf konfrontieren, sie unterbrechen, nicht ausreden lassen und ihre Einwände entkräften (z. B. „Es gibt überhaupt keine Zweifel daran, dass Sie die Tat begangen haben“; Inbau et al. 2013). Um Beschuldigte dazu zu bringen, dass sie die Beweislage überschätzen, sollen Vernehmende sie wiederholt mit Beweisen konfrontieren, die Beweislage übertreiben (z. B. „Wir haben Ihren Namen nicht aus einem Hut gezogen. Sie müssen langsam einsehen, dass es haufenweise Beweise gegen Sie gibt"; Nasher 2015) oder die Beschuldigten gegeneinander ausspielen (z.B. „Wir wissen doch beide, dass in jedem Fall mit mehreren Beteiligten früher oder später einer anfängt zu reden, und in Ihrem Fall sollten Sie derjenige sein"; Inbau et al. 2013). Um zu erreichen, dass Beschuldigte die Konsequenzen von Bestreiten hoch einschätzen, sind auch hier 3 Arten von Maximierungstaktiken zu unterscheiden: (a) Maximierung der moralischen Konsequenzen (z. B. ,Wenn Sie weiter bestreiten, dann kann die Familie des Opfers keine Ruhe finden"); (b) Maximierung der psychologischen Konsequenzen (z.B. „Wenn Sie jetzt nichts sagen, dann denken die Leute, dass Sie so etwas immer machen"; Senese 2012), (c) Maximierung der rechtlichen Konsequenzen (z.B. „Wissen Sie noch, wie es war, als Sie klein waren 
und etwas Schlimmes getan haben? Sie wurdest zwar bestraft, wenn Sie die Wahrheit gesagt haben, aber wenn Sie alles bestritten haben, wurden Sie dann nicht viel härter bestraft?"; Nasher 2015). Auch hier zielen die konfrontativdruckausübenden Vorgehensweisen der Vernehmenden auf eine Zustandsänderung bei den Beschuldigten ab (d.h. verunsichert-aussichtslos fühlen; Beweise hoch einschätzen; Konsequenzen hoch einschätzen), die zu einer Aussage führen soll.

Die Darstellungen in Abb. 1 und 2 mit Zwischen- und Hauptzielen können eine Grundlage bieten, um einzelne Wirkungsweisen auf emotional-kognitiver Ebene und verbaler Ebene (Aussage, Geständnis) zu untersuchen: Zum Beispiel kann untersucht werden, ob Minimierungstaktiken wie freundlich, sympathisch und unaufdringlich sein, dazu führen, dass Beschuldigte (a) sich subjektiv eher sicher fühlen, (b) eher Angaben machen und (c) eher Geständnisse ablegen.

\section{Sozialpsychologische Mechanismen von Geständnissen und manipulativ- minimierende und maximierende Einflussnahmen}

Eine Metaanalyse fand, dass wahre Geständnisse eher aus innerpsychologischen Motivationen (z.B. Schuld oder Reue) heraus entstehen und falsche Geständnisse eher auf äußere Faktoren - wie z.B. Vernehmungstaktiken - zurückzuführen sind (Houston et al. 2014). Deshalb wird nun auf psychologische Mechanismen eingegangen, die die sozialen Einflussnahmen von Minimierung und Maximierung erklären können.

\section{Beeinflussung des Verhaltens durch kurz- und langfristige Konsequenzen}

Menschliches Verhalten wird sehr viel stärker durch unmittelbare als durch langfristige Konsequenzen beeinflusst (z. B. Björkman 1984). Personen bevorzugen beispielsweise spätere Bestrafung im Vergleich zu unmittelbarer Bestrafung, da sie ihr aktuelles Wohlbefinden maximieren wollen (Renner 1964). Außerdem wählen laut der ProspectTheorie von Kahneman und Tversky (1979) Personen eher Entscheidungsalternativen, die sicher sind, als wahrscheinliche Alternativen. Dabei werden unmittelbare Konsequenzen als sicherer eingestuft als langfristige Konsequenzen (Kalenscher und Pennartz 2008). Auch das Entscheidungsmodell bei Geständnissen (,,decision-making model of confessions") von Hilgendorf und Irving (1981) nimmt an, dass kurzfristige Konsequenzen einen stärkeren Einfluss auf Geständnisentscheidungen haben als langfristige Konsequenzen. Die Entscheidungen in einer Vernehmung sind dabei von den subjektiven Einschätzungen dieser Konsequenzen abhängig (und nicht von den realistischen). Oftmals haben Beschuldigte jedoch nur wenig fundierte Informationen $\mathrm{zu}$ den Konsequenzen. Laut diesem Modell gestehen Unschuldige, um die Vernehmung zu beenden (kurzfristige positive Konsequenz) und weil sie gleichzeitig davon ausgehen, dass im weiteren Verlauf der Ermittlungen ihre Unschuld bewiesen wird. Dabei werden persönliche Konsequenzen oft mehr gefürchtet als juristische, da erstere kurzfristiger auftreten (St-Yves und Deslauriers-Varin 2009). Auch Gudjonsson (2003) nimmt an, dass unschuldige Beschuldigte sich der langfristigen Konsequenzen teilweise oder völlig bewusst sind, aber gleichzeitig zuversichtlich sind, dass sich ihre Unschuld später zeigen wird. Im Einklang damit fanden zwei Experimente, dass Personen eher ein Geständnis ablegten, um kurzfristigen negativen Konsequenzen zu entgehen, obwohl sie damit gleichzeitig das Risiko langfristiger negativer Konsequenzen erhöhten (Madon et al. 2012, 2013). Dies erklärt, warum manche unschuldige Beschuldigte die unangenehme Vernehmungssituation mit einem Geständnis beenden wollen, obwohl dies das Risiko einer späteren Verurteilung erhöht (Sigurdsson und Gudjonsson 1996). Dieses Risiko missachtet jedoch das Reid-Manual: Denn demnach ist das oberste Ziel einer Vernehmung, Beschuldigte davon abzuhalten, sich über die langfristigen negativen Konsequenzen eines Geständnisses Gedanken zu machen (Inbau et al. 2013).

Die subjektive Einschätzung der Konsequenzen und des Nutzens eines Geständnisses kann von außen (z.B. durch Vernehmende) beeinflusst werden, sodass eine Geständnisentscheidung begünstigt wird (Hilgendorf und Irving 1981). So kann es durch Vorgehensweisen, die die Tatverantwortung herunterspielen (,Das Opfer hat die Tat sicherlich übertrieben dargestellt"; Abb. 1: Tatverantwortung gering einschätzen) dazukommen, dass Beschuldigte die negativen langfristigen rechtlichen Konsequenzen eines Geständnisses als geringer betrachten, als sie es eigentlich sind. Auch in einer Vignettenstudie zeigte sich, dass eine solche Vorgehensweise zu einer geringeren Straferwartung führte (Kassin und McNall 1991). Ebenso werden durch Aussagen von Vernehmenden wie „Sie werden sich nach dem Geständnis besser fühlen“ (Abb. 1: psychologische Konsequenzen gering einschätzen) unmittelbare positive Konsequenzen in Aussicht gestellt. Eine implizite Minimierung der Konsequenzen eines Geständnisses kann dabei von Beschuldigten als Versprechen verstanden werden, dass ein Geständnis zu Strafmilderungen führt (Kassin und McNall 1991). Dies lässt sich mit dem Phänomen der sog. pragmatischen Implikation beschreiben, wonach bei fehlenden expliziten Versprechungen Personen überlegen, interpretieren und schlussfolgern, welche Konsequenzen zu erwarten sind (Chan und McDermott 2006; Harris und Monaco 1978; Hilton 1995). Oft werden dann nur die pragmatischen Schluss- 
folgerungen gespeichert und erinnert und nicht das, was eigentlich gesagt wurde. Im Einklang damit fanden Luke und Alceste (2020), dass Versuchspersonen eine geringere Straferwartung hatten, wenn in einem Ermittlungsbericht mit Vernehmungsprotokoll a) die Tat moralisch heruntergespielt wurde oder b) explizite Versprechungen gemacht wurden (im Vergleich zu einer Kontrollbedingung). Redlich et al. (2020) kamen zu ähnlichen Ergebnissen: Versuchspersonen, die eine Fallbeschreibung mit einem expliziten oder einem impliziten Versprechen auf Strafmilderung gelesen hatten, gaben dreimal häufiger an, dass der Beschuldigte gestehen sollte als in der Kontrollbedingung.

Maximierungstaktiken hingegen können dazu führen, dass Personen die Vernehmung als sehr unangenehm empfinden und diese deswegen unmittelbar mittels eines Geständnisses beenden wollen. So können Vernehmende beispielsweise Beschuldigte wiederholt mit dem Tatvorwurf konfrontieren, diese nicht ausreden lassen und alle Einwände entkräften (z. B. „Lassen Sie mich ausreden. Wir haben überhaupt keine Zweifel, dass Sie die Tat begangen haben"; Abb. 2: verunsichert und aussichtslos fühlen), was $\mathrm{zu}$ einer unangenehmen Vernehmungssituation führt. Im Einklang mit dem Entscheidungsmodell kann es in solchen Fällen durch Maximierungstaktiken dann noch dazu kommen, dass Beschuldigte die negativen Konsequenzen von anhaltendem Bestreiten überschätzen (z. B. ,Wenn Sie weiter sagen, Sie waren es nicht, kann ich nichts mehr für Sie tun“; Abb. 2: rechtliche Konsequenzen hoch einschätzen) und Gestehen als einzige Option wahrnehmen (Hilgendorf und Irving 1981).

Zusammenfassend können also Minimierung und Maximierung die Wahrscheinlichkeit von Geständnissen erhöhen, indem unmittelbare positive Konsequenzen eines Geständnisses in Aussicht gestellt werden und gleichzeitig negative Konsequenzen von Bestreiten übertrieben werden. Dies ist jedoch besonders gefährlich, da - wie von Horgan et al. (2012) gefunden und oben ausgeführt - bei Manipulation der Konsequenzen die Wahrscheinlichkeit falscher Geständnisse zunimmt und gleichzeitig von wahren Geständnissen abnimmt.

\section{Konditionierung von Verhalten durch Belohnung und Bestrafung}

Allgemein reagieren Menschen und daher auch Beschuldigte sehr stark auf Verstärkung und Bestrafung (z. B. Herrnstein 1970; Kassin 2007). Mittels (operanter) Konditionierung sind Verhaltensweisen durch Bestrafung und Verstärkung erlernbar (Häcker und Stapf 2004): Erwünschtes Verhalten kann durch positive Verstärkung (Hinzufügen eines positiven Reizes; z.B. Anerkennung, Lob) und negative Verstärkung aufgebaut werden (Entfernung eines negativen Reizes; z. B. Beenden der Vernehmungssituation). Mittels positiver Bestrafung (Hinzufügen eines negativen Reizes; z.B. Beschimpfungen) und negativer Bestrafung (Entfernung eines positiven Reizes; z.B. Entzug von Aufmerksamkeit) können Verhaltensweisen verlernt und abgebaut werden.

Im Vernehmungskontext können Vernehmende durch Minimierung und Maximierung Verhalten verstärken und bestrafen und dadurch Entscheidungen zum Aussageverhalten von Beschuldigten beeinflussen. Loben beispielsweise Vernehmende Beschuldigte für Einlassungen und zeigen sich ihnen anerkennend, kann sich das positiv verstärkend auf die Beschuldigten auswirken, sodass sie sich in Sicherheit fühlen, Aussagen machen und eher Geständnisse ablegen (Abb. 1). Positiv bestrafende Vorgehensweisen der Vernehmenden sind beispielsweise, dass sie bestreitende Beschuldigte beschimpfen oder wiederholt als Lügner bezeichnen. Dies könnte - im Sinne der positiven Bestrafung - dazu führen, dass sich Beschuldigte verunsichertaussichtslos fühlen und deshalb eher Aussagen machen und so die Wahrscheinlichkeit von Geständnissen erhöht wird (Abb. 2). Besonders problematisch ist dies, wenn auf anhaltendes Bestreiten von unschuldig Beschuldigten durch schuldüberzeugte Vernehmende mit einer verstärkten Anwendung von Maximierung geantwortet wird. Solch verstärkende und bestrafende Elemente können einerseits die Wahrscheinlichkeit falscher Geständnisse erhöhen, aber - wie von Gudjonsson (1995) anhand von Fallbeispielen diskutiert - auch zu einem „Boomerang-Effekt“ führen (d.h. Vernehmungsdruck führt bei Beschuldigten dazu, dass sie die entgegengesetzte Sichtweise einnehmen und beispielsweise eine geständige Einlassung zurückziehen).

\section{Beeinflussung durch Autoritätspersonen}

Klassische Experimente zeigten, dass Menschen sozial beeinflussbar und gehorsam gegenüber Autoritätspersonen sind (z.B. Milgram 1963). Im Vernehmungskontext sind Vernehmende als Autoritätsfiguren zu betrachten, die über Macht gegenüber den Beschuldigten verfügen. Beispielsweise entscheiden in der Regel Vernehmende, ob bzw. wann die Vernehmung unterbrochen oder beendet wird.

Maximierungstaktiken enthalten autoritäre Verhaltensweisen der Vernehmenden wie beispielsweise, Beschuldigte wiederholt mit Tatvorwürfen zu konfrontieren oder mit absoluter Sicherheit zu behaupten, dass sie die Tat begangen haben. Solche Taktiken können dazu führen, dass Beschuldigte sich verunsichert-aussichtslos fühlen und deshalb Aussagen machen (Abb. 2). Auch das Entscheidungsmodell nimmt an, dass autoritäres Vorgehen zu Gehorsam und falschen Geständnissen bei unschuldigen Beschuldigten führen kann (Hilgendorf und Irving 1981).

Autoritätspersonen können außerdem im Sinne einer „Beraterfunktion“ Einfluss auf andere Personen neh- 
men. Minimierungstaktiken enthalten solche beratenden Elemente: So sind Beschuldigte, die sich freundlich/ freundschaftlich von Vernehmenden behandelt fühlen und ihnen vertrauen (d.h. in Sicherheit fühlen) vermutlich offener für Ratschläge und Empfehlungen. Solche „freundschaftlichen Beratungen“ durch eine Autoritätsperson haben vermutlich einen sehr viel stärkeren Einfluss auf die Entscheidungen und Aussagen von Beschuldigten als Beratungen durch Nicht-Autoritätspersonen (z.B. da polizeilich Vernehmende sich in der Regel mit strafrechtlichen Konsequenzen besser auskennen als sie selbst).

Oftmals treten viele der hier beschriebenen sozialpsychologischen Mechanismen in Vernehmungen zusammen auf. So kann beispielsweise der Einfluss von beratenden Autoritätspersonen verstärkt werden, da die erwarteten negativen Konsequenzen eines Geständnisses verzögert eintreten und gleichzeitig Beschuldigte aufgrund impliziter Versprechen eine Strafmilderung bei einem Geständnisses annehmen. Auch Milgram (1963) fand, dass die Tendenz zu Gehorsam verstärkt wurde, wenn die negativen Konsequenzen zeitlich oder räumlich eine größere Distanz aufwiesen. Im Einklang damit kann es dazukommen, dass unschuldige Beschuldigte in einer Vernehmungssituation ein falsches Geständnis ablegen und gleichzeitig davon ausgehen, dass sich ihre Unschuld im weiteren Verlauf eines Strafverfahrens zeigen wird.

\section{Schlussfolgerungen}

Minimierungs- und Maximierungstaktiken haben als Hauptziel, ein Geständnis von Beschuldigten zu erlangen. Hierfür werden verschiedene Vorgehensweisen angewendet, die einerseits die Tat und Tatfolgen herunterspielen und zu einem Gefühl der Sicherheit führen und andererseits die Folgen des Bestreitens einer Straftat maximieren und eine Verunsicherung fördern. Beide Taktiken sind somit manipulativ, verfolgen das Zwischenziel, den subjektiven Zustand bei Beschuldigten zu ändern und den Aussagewiderstand aufzulösen und darauf aufbauend ein Geständnis zu erzielen. Experimente zeigen, dass Minimierung und Maximierung die Wahrscheinlichkeit falscher Geständnisse erheblich erhöhen. Trotzdem empfehlen Vernehmungsmanuale, die vornehmlich in den USA entwickelt wurden, und einzelne deutschsprachige Veröffentlichung die Anwendungen dieser Taktiken. Schriftstücke und Einzelfälle weisen darauf hin, dass auch die Polizei in Deutschland teilweise in solchen Taktiken geschult wurde und diese angewendet hat.

Inwiefern einzelne Arten von Minimierung und Maximierung die subjektiven Einschätzungen und das Aussageverhalten von Beschuldigten beeinflussen, wurde bisher nicht differenziert untersucht. Vielmehr wurde der Einfluss von Kombinationen aus verschiedenen Vorgehensweisen auf Geständnisse beleuchtet. Differenzierte Erkenntnisse zu den Effekten der Taktiken sind jedoch notwendig, um ihre Folgen besser einschätzen und erklären zu können. Die in dieser Arbeit präsentierten Systematisierungen und sozialpsychologischen Erklärungen können helfen, zukünftig Wirkungsweisen einzelner Vorgehensweisen von Minimierungs- und Maximierungstaktiken auf Einschätzungen, Aussagemotivation und Geständnisrate differenzierter zu untersuchen, besser zu verstehen und theoretisch zu begründen.

International hat teilweise ein Umdenken stattgefunden (Snook et al. 2020). Beispielsweise haben sich Unternehmen wie Wicklander und Zulawski (www.w-Z.com) von konfrontativ-druckausübenden Befragungsseminaren wegbewegt und selbst John E. Reid and Associates, Inc. bietet nun nichtdruckausübende Fortbildungsseminare an (www. reid.com). Snook et al. (2020) beschreiben jedoch, dass dies in Kanada zu einem „Werkzeugkasten“ bei Ermittelnden führt: In diesen nehmen sie wissenschaftlich-fundierte und effektive Vernehmungstaktiken auf, wenn sie ihren Bedürfnissen entsprechen; jedoch greifen sie auch weiterhin bei Bedarf auf Taktiken wie Minimierung und Maximierung zurück. Die subjektive Notwendigkeit zum Rückgriff auf diese Taktiken dürfte besonders dann vorliegen, wenn Vernehmende von der Schuld der Beschuldigten überzeugt sind, weitere belastende Beweise fehlen und sie mit einem offenen Vernehmungsvorgehen kein Geständnis erzielen können (May 2021). Dabei scheint es zu einer Abnahme in der Anwendung von Maximierungstaktiken zu kommen, wohingegen Minimierungstaktiken weiter angewendet werden (Snook et al. 2020). Ähnliche Muster ergeben sich teilweise auch in Gesprächen mit Polizist*innen aus Deutschland. Ein solches Vorgehen lässt sich durch die hier skizzierten Forschungsarbeiten nicht begründen und ist vielmehr damit zu erklären, dass das Manipulationspotenzial und das Risiko falscher Geständnisse bei Minimierung unterschätzt werden (Kassin und McNall 1991).

Vor diesem Hintergrund wäre es wünschenswert, dass Polizist*innen sowie Psycholog*innen zukünftig eng zusammenarbeiten, um die Vernehmungsqualität in Deutschland zu erhöhen, wie es in anderen Ländern schon länger der Fall ist (z.B. Großbritannien, Norwegen). Dabei sollten die umfangreichen internationalen Forschungsarbeiten zu Vernehmungen und Aussagen von Beschuldigten, die eine fundierte Wissensgrundlage erarbeitet haben, beachtet werden. Vernehmungstechniken, die zu zuverlässigen Informationen führen, müssen für den deutschsprachigen Raum somit nicht von Grund auf neu entwickelt werden. Vielmehr können systematische Vernehmungstrainings (z.B. Reinhold et al. 2021) zur Erhebung zuverlässiger Informationen auf international wissenschaftlich fundierte Vorgehensweisen (z. B. zum Freien Bericht; Lamb und Brown 2018; 
Wilson und Powell 2001) und Modelle (z. B. das PEACEModell, Clarke und Milne 2001; das CTI Training Tool: Investigative Interviewing for Criminal Cases [eine deutsche Übersetzung ist abrufbar], Convention Against Torture Initiative 2017 oder die Principles on Effective Interviewing for Investigations and Information Gathering, Association for the Prevention of Torture 2021) zurückgreifen und so die Vernehmungsqualität schrittweise verbessern.

Funding Open Access funding enabled and organized by Projekt DEAL.

Interessenkonflikt T. Schneider und L. May geben an, dass kein Interessenkonflikt besteht.

Open Access Dieser Artikel wird unter der Creative Commons Namensnennung 4.0 International Lizenz veröffentlicht, welche die Nutzung, Vervielfältigung, Bearbeitung, Verbreitung und Wiedergabe in jeglichem Medium und Format erlaubt, sofern Sie den/die ursprünglichen Autor(en) und die Quelle ordnungsgemäß nennen, einen Link zur Creative Commons Lizenz beifügen und angeben, ob Änderungen vorgenommen wurden.

Die in diesem Artikel enthaltenen Bilder und sonstiges Drittmaterial unterliegen ebenfalls der genannten Creative Commons Lizenz, sofern sich aus der Abbildungslegende nichts anderes ergibt. Sofern das betreffende Material nicht unter der genannten Creative Commons Lizenz steht und die betreffende Handlung nicht nach gesetzlichen Vorschriften erlaubt ist, ist für die oben aufgeführten Weiterverwendungen des Materials die Einwilligung des jeweiligen Rechteinhabers einzuholen.

Weitere Details zur Lizenz entnehmen Sie bitte der Lizenzinformation auf http://creativecommons.org/licenses/by/4.0/deed.de.

\section{Literatur}

Artkämper H, Schilling K (2018) Vernehmungen: Taktik, Psychologie, Recht. Deutsche Polizeiliteratur

Association for the Prevention of Torture (2021) Principles on effective interviewing for investigations and information gathering. https:// www.apt.ch/en/resources/publications/new-principles-effectiveinterviewing-investigations-and-information. Zugegriffen: 4. Juni 2021

Bayerischer Landtag (2014) Schriftliche Anfrage des Abgeordneten Florian Ritter SPD vom 04.06.2014 Ausbildung in und Nutzung der REID-Methode durch bayerische Behörden, insbesondere bei den Ermittlungen zur Ceska-Mordserie. https://www. bayern.landtag.de/www/ElanTextAblage_WP17/Drucksachen/ Schriftliche\%20Anfragen/17_0002778.pdf. Zugegriffen: 4. Juni 2021

Björkman M (1984) Decision making, risk taking and psychological time: review of empirical findings and psychological theory. Scand J Psychol 25(1):31-49. https://doi.org/10.1111/j.14679450.1984.tb00999.x

Bundesregierung (2014). Drucksache 18/1413. Antwort der Bundesregierung auf die Kleine Anfrage der Abgeordneten Martina Renner, Wolfgang Gehrcke, Annette Groth, weiterer Abgeordneter und der Fraktion DIE LINKE. Ausbildung in und Nutzung der Reid-Methode durch deutsche Bundesbehörden. http://dip21. bundestag.de/dip21/btd/18/014/1801413.pdf

Chan JCK, McDermott KB (2006) Remembering pragmatic inferences. Appl Cognit Psychol 20(5):633-639. https://doi.org/10. 1002/acp. 1215

Clarke C, Milne B (2001) National evaluation of the PEACE investigative interviewing course. PRAS; No. 149
Convention Against Torture Initiative (2017) CTI Training Tool 1/2017: Die Untersuchende Vernehmungstechnik [Investigative Interviewing for Criminal Cases. Prepared by the Norwegian Center for Human Rights (NCHR) in cooperation with the Norwegian Police University College]. https://cti2024.org/resource/ctitraining-tool-1-2017-investigative-interviewing-for-criminalcases-german-die-untersuchende-vernehmungstechnik/. Zugegriffen: 4. Juni 2021

DePaulo BM, Lindsay JJ, Malone BE, Muhlenbruck L, Charlton K, Cooper H (2003) Cues to deception. Psychol Bull 129(1):74-118. https://doi.org/10.1037/0033-2909.129.1.74

Drews F (2012) Die Königin unter den Beweismitteln? Eine interdisziplinäre Untersuchung des (falschen) Geständnisses. LIT

Findley KA, Scott MS (2006) The multiple dimensions of tunnel vision in criminal cases. Wis L Rev 2:291-397

Gubi-Kelm S, Grolig T, Strobel B, Ohlig S, Schmidt AF (2020) When do false accusations lead to false confessions? Preliminary evidence for a potentially overlooked alternative explanation. J Forensic Psychol Res Pract 20(2):114-133. https://doi.org/10.1080/ 24732850.2020 .1714388

Gudjonsson GH (1995) The effects of interrogative pressure on strategic coping. Psychol Crime Law 1(4):309-318. https://doi.org/10. 1080/10683169508411968

Gudjonsson GH (2003) The psychology of interrogations and confessions: A handbook. John Wiley \& Sons

Häcker H, Stapf KH (2004) Dorsch Psychologisches Wörterbuch. Huber

Harris RJ, Monaco GE (1978) Psychology of pragmatic implication: Information processing between the lines. J Exp Psychol Gen 107(1):1-22. https://doi.org/10.1037/0096-3445.107.1.1

Hermanutz M, Litzcke S (2012) Vernehmung in Theorie und Praxis. Wahrheit - Irrtum - Lüge. Richard Boorberg

Herrnstein RJ (1970) On the law of effect. J Exp Anal Behav 13(2):243-266. https://doi.org/10.1901/jeab.1970.13-243

Heubrock D, Donzelmann N (2010) Psychologie der Vernehmung. Empfehlungen zur Beschuldigten-, Zeugen- und OpferzeugenVernehmung. Verlag für Polizeiwissenschaft

Hilgendorf EL, Irving B (1981) A decision-making model of confessions. In: Lloyd-Bostock MA (Hrsg) Psychology in legal contexts. Applications and limitations. MacMillan, S 67-84

Hilton DJ (1995) The social context of reasoning: conversational inference and rational judgment. Psychol Bull 118(2):248-271. https://doi.org/10.1037/0033-2909.118.2.248

Horgan AJ, Russano MB, Meissner CA, Evans JR (2012) Minimization and maximization techniques: assessing the perceived consequences of confessing and confession diagnosticity. Psychol Crime Law 18(1):65-78. https://doi.org/10.1080/1068316x. 2011.561801

Houston KA, Meissner CA, Evans JR (2014) Psychological processes underlying true and false confessions. In: Bull R (Hrsg) Investigative Interviewing. Springer, Berlin, S 19-34

Inbau FE, Reid JE, Buckley JP, Jayne BC (2013) Essentials of the Reid technique: Criminal interrogation and confessions. Jones \& Bartlett

Jung I, Lemmer C (2013) Der Fall Peggy. Die Geschichte eines Skandals. Droemer

Kahneman D, Tversky A (1979) Prospect theory of decisions under risk. Econometrica 47(2):263-291. https://doi.org/10.2307/ 1914185

Kalenscher T, Pennartz CMA (2008) Is a bird in the hand worth two in the future? The neuroeconomics of intertemporal decisionmaking. Prog Neurobiol 84(3):284-315. https://doi.org/10.1016/ j.pneurobio.2007.11.004

Kassin SM (2007) Expert testimony on the psychology of confessions: a pyramidal framework of the relevant science. In: Borgida E, Fiske ST (Hrsg) Beyond common sense: psychological science in the courtroom. Blackwell, S 195-218 
Kassin SM, Kiechel KL (1996) The social psychology of false confessions: compliance, internalization, and confabulation. Psychol Sci 7(3):125-128. https://doi.org/10.1111/j.1467-9280.1996. tb00344.X

Kassin SM, McNall K (1991) Police interrogations and confessions. Law Hum Behav 15(3):233-251. https://doi.org/10.1007/ bf01061711

Kassin SM, Neumann K (1997) On the power of confession evidence: an experimental test of the fundamental difference hypothesis. Law Hum Behav 21(5):469-484. https://doi.org/10.1023/a: 1024871622490

Kassin SM, Bogart D, Kerner J (2012) Confessions that corrupt: evidence from the DNA exoneration case files. Psychol Sci 23(1):41-45. https://doi.org/10.1177/0956797611422918

Kassin SM, Drizin SA, Grisso T, Gudjonsson GH, Leo RA, Redlich AD (2010) Police-induced confessions: risk factors and recommendations. Law Hum Behav 34(1):3-38. https://doi.org/ 10.1007/s10979-009-9188-6

Kassin SM, Fong CT (1999) "I'm innocent!": Effects of training on judgments of truth and deception in the interrogation room. Law Hum Behav 23(5):499-516. https://doi.org/10.1023/a:102233001

Klaver JR, Lee Z, Rose VG (2008) Effects of personality, interrogation techniques and plausibility in an experimental false confession paradigm. Leg Criminol Psychol 13(1):71-88. https://doi.org/10. 1348/135532507x 193051

Lamb ME, Brown DA (2018) Tell me what happened: questioning children about abuse. Wiley-Blackwell

Leo RA (1996) Inside the interrogation room. J Crim Law Criminol 86(2):266-303. https://doi.org/10.2307/1144028

Leo RA (2020) Structural police deception in american police interrogation: a closer look at minimization and maximization. In: Eidam L, Lindemann M, Ransiek A (Hrsg) Interrogation confession and truth: comparative studies in criminal procedure. Nomos, S $183-207$

Lindner J-E (2002) Polizeipräsident will mit unechten Beweisen Druck ausüben. Verhör mit Psycho-Tricks. Hamburger Abendblatt. https://www.abendblatt.de/hamburg/article107025567/Verhoermit-Psycho-Tricks.html. Zugegriffen: 1. Feb 2021

Luke TJ, Alceste F (2020) The mechanisms of minimization: how interrogation tactics suggest lenient sentencing through pragmatic implication. Law Hum Behav 44(4):266-285. https://doi.org/10. 1037/lhb0000410

Luther K, Snook B (2016) Putting the mr. Big technique back on trial: a re-examination of probative value and abuse of process through a scientific lens. J Forensic Pract 18(2):131-142. https://doi.org/ 10.1108/JFP-01-2015-0004

Madon S, Guyll M, Scherr KC, Greathouse S, Wells GL (2012) Temporal discounting: the differential effect of proximal and distal consequences on confession decisions. Law Hum Behav 36(1):13-20. https://doi.org/10.1037/h0093962

Madon S, Yang Y, Smalarz L, Guyll M, Scherr KC (2013) How factors present during the immediate interrogation situation produce short-sighted confession decisions. Law Hum Behav 37(1):60-74. https://doi.org/10.1037/lhb0000011

May L (2021) Das Risiko unzuverlässiger Informationen in unternehmensinternen Befragungen und Vernehmungen: eine psychologische Perspektive. In Artkämper H, Floren T, Schilling K (Hrsg) Vernehmungen: Taktik - Psychologie - Recht. Verlag Deutsche Polizeiliteratur, S 567-614

May L (2019) Verhöre, Vernehmungen und Befragungen: Was für Aussagen sind zu erwarten? In: Bischoff C, Juwig C, Sommer L (Hrsg) Bekenntnisse: Formen und Formeln. Reimer, S 101-118

Meissner CA, Russano MB, Narchet FM (2010) The importance of a laboratory science for improving the diagnostic value of confession evidence. In: Lassiter GD, Meissner C (Hrsg) Police interrogations and false confessions: current research, practice, and policy recommendations. APA, S 111-126
Milgram S (1963) Behavioral study of obedience. J Abnorm Soc Psychol 67(4):371-378. https://doi.org/10.1037/h0040525

Napier MR, Adams SH (1998) Magic words to obtain confessions. FBI Law Enforc 67(10):11-15

Narchet FM, Meissner CA, Russano MB (2011) Modeling the influence of investigator bias on the elicitation of true and false confessions. Law Hum Behav 35(6):452-465. https://doi.org/10.1007/ s10979-010-9257-x

Nasher J (2015) Entlarvt! Wie Sie in jedem Gespräch an die ganze Wahrheit kommen. Goldmann

Redlich AD, Shteynberg RV, Nirider LH (2020) Pragmatic implication in the interrogation room: a comparison of juveniles and adults. J Exp Criminol 16:555-564. https://doi.org/10.1007/s11292-01909377-y

Reinhold S, Yoon D, May L (2021) Das interaktive Vernehmungstraining (invetra) - ein Training zum Erlernen eines psychologisch angemessenen und effektiven Vernehmungsvorgehens? Kriminalistik 5:289-295

Renner KE (1964) Delay of reinforcement: a historical review. Psychol Bull 61(5):341-361. https://doi.org/10.1037/h0048335

Rick R (2012) An die Hunde verfüttert - Prozessbericht zu einem Justizirrtum. Strafverteidiger Forum 10:400-405

Russano MB, Meissner CA, Narchet FM, Kassin SM (2005) Investigating true and false confessions within a novel experimental paradigm. Psychol Sci 16(6):481-486. https://doi.org/10.1111/j.09567976.2005.01560.x

Scherr KC, Redlich AD, Kassin SM (2020) Cumulative disadvantage: a psychological framework for understanding how innocence can lead to confession, wrongful conviction, and beyond. Perspect Psychol Sci 15(2):353-383. https://doi.org/10.1177/ 1745691619896608

Schneider T, Sauerland M, Merckelbach H, Puschke J, Cohrs JC (2021) Voluntary blame-taking behavior: Kinship before friendship and no effect of incentives. Front Psychol Leg Psychol. 12: Article 621960. https://doi.org/10.3389/fpsyg.2021.621960

Senese LC (2012) Anatomy of interrogation themes. John E. Reid and Associates

Sigurdsson JF, Gudjonsson GH (1996) The psychological characteristics of false confessors. A study among Icelandic prison inmates and juvenile offenders. Pers Individ Dif 20(3):321-329. https:// doi.org/10.1016/0191-8869(95)00184-0

Smith SM, Stinson V, Patry MW (2009) Using the "mr. Big" technique to elicit confessions: successful innovation or dangerous development in the Canadian legal system? Psychol Public Policy Law 15(3):168-193. https://doi.org/10.1037/a0016962

Smith SM, Stinson V, Patry MW (2010) High-risk interrogation: using the "Mr. Big Technique" to elicit confessions. Law Hum Behav 34(1):39-40. https://doi.org/10.1007/s10979-009-9203-y

Snook B, Fahmy W, Fallon L, Lively CJ, Luther K, Meissner CA, Barron T, House JC (2020) Challenges of a "toolbox" approach to investigative interviewing: a critical analysis of the Royal Canadian Mounted Police's (RCMP) Phased Interview Model. Psychol Public Policy Law 26(3):261-273. https://doi.org/10.1037/ law0000245

St-Yves M, Deslauriers-Varin N (2009) The psychology of suspects' decision-making during interrogation. In: Bull $R$, Valentine $T$, Williamson T (Hrsg) Handbook of psychology of investigative interviewing: current developments and future directions https:// doi.org/10.1002/9780470747599.ch1

van Toor DAG, Horselenberg RR (2020) The mr. Big method: on or beyond the boundaries of lawfulness and reliability? In: Eidam L, Lindemann M, Ransiek A (Hrsg) Interrogation, Confession, and Truth, S 87-122 https://doi.org/10.5771/9783748904380-87

Volbert R, May L (2016) Falsche Geständnisse in polizeilichen Vernehmungen - Vernehmungsfehler oder immanente Gefahr. Recht Psychiatr 34:4-10

Volbert R, May L, Hausam J, Lau S (2019) Confessions and denials when guilty and innocent: forensic patients' self-reported be- 
havior during police interviews. Front Psychiatry 10:Article 168. https://doi.org/10.3389/fpsyt.2019.00168

Vrij A, Hartwig M, Granhag PA (2019) Reading lies: nonverbal communication and deception. Annu Rev Psychol 70(1):295-317. https://doi.org/10.1146/annurev-psych-010418-103135

Vrij A, Mann S, Fisher RP (2006) An empirical test of the behaviour analysis interview. Law Hum Behav 30(3):329-345. https://doi. org/10.1007/s10979-006-9014-3
Wilson JC, Powell MB (2001) A guide to interviewing children: essential skills for counsellors, police, lawyers and social workers. Unwin

Wirth I (2013) Kriminalistik-Lexikon. Kriminalistik 